\title{
Libro: La Política en tiempos de los Kirchner \\ Andrés Malamud y Miguel De Luca (coordinadores)
}

Eudeba, Buenos Aires, 2011 (336 pp.)

\section{Julieta Zelicovich}

Licenciada en Relaciones Internacionales y becaria doctoral del Consejo Nacional de Investigaciones Científicas y Técnicas, en la Facultad de Ciencia Política y Relaciones Internacionales, Universidad Nacional de Rosario, Argentina.

E-mail: jzelicovich@yahoo.com.ar

La finalización del primer mandato de Cristina Fernández de Kirchner constituye un buen hito para convocar a la ciencia política a la reflexión sobre los cambios y continuidades que las administraciones iniciadas en 2003 han supuesto para la historia y praxis política argentina. Así parecen entenderlo Malamud y De Luca en La Política en tiempos de los Kirchner. Como se enuncia en el prefacio de la obra, "tras ocho años en el poder, el kirchnerismo ha producido suficiente 'realidad' como para ser interpelada analítica y sistemáticamente desde la ciencia política".

El libro presenta 23 capítulos, agrupados en 4 secciones, escritos por diversos politólogos que comparten un pasado y/o presente en la Universidad de Buenos Aires, y un conjunto de interrogantes en común, a saber: ¿Qué cambió entre 2003 y 2011? ¿Y que no cambió? ¿Cuáles fueron los actores centrales y los hitos que marcaron esta etapa? ¿Qué perspectivas se avizoran para el futuro?

Sin caer en tecnicismos, la obra se distingue por su contenido específico de análisis político, y por presentar una perspectiva pluralista de las administraciones bajo estudio. Se destaca también por incluir una preocupación por contextualizar las reflexiones, existiendo permanentes referencias a períodos anteriores. A pesar de las aclaraciones introductorias sorprende, quizás, la ausencia de un capítulo dedicado a la política económica, siendo éste un aspecto relevante en el período analizado. De todos modos, tal inquietud del lector puede ser parcialmente salvada por la mención, en varios capítulos, de temas como la política tributaria o la política financiera.

El concepto de "arquitectura de poder", utilizado por Tonelli en la introducción, y referido a la forma de ejercicio del poder y organización del Poder Ejecutivo, sirve como articulador para gran parte del libro. De forma recurrente, se caracterizará a ambas administraciones por su estilo centralizado y personalista de ejercer el poder, y por su bajo interés por la construcción institucional, reflejándose ello en cuestiones diversas tales como la relación entre el gobierno nacional y las provincias, la construcción del discurso y de la estrategia de comunicación, o bien, la gestión de la política externa.

Si bien ambas administraciones son concebidas como continuación una de la otra, los autores hacen énfasis en la existencia de un momento de cambio y/o ajuste en las políticas analizadas. Así el "conflicto con el campo" sobresale como un hecho que cambió de forma definitiva al kirchnerismo. En lo institucional, con la 
ruptura de éste con la Concertación Plural y con el vicepresidente Cobos; y con el cambio en las perspectivas de mediano plazo dentro del Congreso. En lo cultural, con la emergencia de Carta Abierta y el enfrentamiento con los "multimedios". En términos globales se trató, como plantea Novaro, de un proceso de "peronización del kirchnerismo".

Otros elementos que atraviesan los capítulos son, justamente, el debate acerca de la identidad peronista-kirchnerista; la importancia del contexto para explicar la evolución de determinadas políticas, y una idea de la democracia como espacio de confrontación o conflicto.

El rasgo hegemónico o cuasi hegemónico en el ejercicio del poder es analizado a lo largo de la primer sección, "Instituciones de Gobierno". En la relación entre presidente y vicepresidente Serrafero señala, para los casos Kirchner-Scioli y Fernández-Cobos, cómo a partir de tal característica, paradójicamente, el papel relegado que adoptaron las vicepresidencias les dio una visibilidad pública que no tenían, y las puso en la agenda del debate. En la conformación y funcionamiento de los gabinetes dicho rasgo hegemónico también estuvo presente. De Luca desarrolla las diferencias de los gabinetes kirchneristas respecto de la tradición justicialista y afirma que la concentración de la toma de decisiones en la cima del poder ejecutivo dio lugar a una relación con el gabinete de tipo radial, y condujo a la limitación del entorno íntimo de la presidencia a un círculo de confianza reducido. En cuanto al funcionamiento del Congreso Nacional, Jones y Micozzi explican, en el análisis de la composición partidaria de las cámaras, parte del porqué de tales rasgos en el ejercicio de la presidencia: sostienen que los períodos con mayoría absoluta posibilitaron al kirchnerismo la decisión de gobernar solo, dado que los números así lo permitían, y también lo hacía la fragmentación de la oposición. Concluye la sección con el análisis de Gargarella acerca de la relación sobre kirchnerismo y justicia, y la identificación de dos etapas: una más liberal-republicana, expresada en la decisión de cambiar la composición de la Corte Suprema, y otra más conservadora, "el giro antiliberal", reflejada en las modificaciones sobre el Consejo de la Magistratura.

En los capítulos de la sección "Actores y Procesos Políticos" cobra relevancia el estudio de los partidos políticos. Tula y De Luca se centran en la evolución del sistema de partidos y en cómo dentro de éstos se produjo la selección de los candidatos a lo largo de la última década. Carrizo por su parte aborda la relación entre presidencialismo y dinámica intra-partidaria. El capítulo de Malamud se aboca al bipartidismo, sosteniendo la continuidad sistémica de los dos principales partidos, radicales y peronistas, en el ordenamiento de la política, aunque de forma asimétrica, situando dicha asimetría en la diferente capacidad de movilización de masas.

Otros trabajos incluidos son el de Zelanik, que aborda las "coaliciones kirchneristas": sociales, electorales y legislativas, y señala el carácter innovador de las primeras, con la incorporación de actores nuevos junto con los tradicionales: los organismos de $\mathrm{DDHH}$, los movimientos piqueteros y el movimiento obrero organizado. También son considerados novedosos algunos aspectos de la relación en- 
tre política subnacional y política nacional, estudiados por Gervasoni, tales como la centralización del federalismo fiscal, la mayor subordinación de las provincias a los gobiernos kirchneristas, incluidos aquellos gobernadores radicales, y la "territorialización" de la política. Estos rasgos contribuyen a reforzar la hipótesis de debilidad institucional presente en este y otros capítulos.

En el cierre de esta sección, Novaro presenta el análisis de la cultura política bajo el kirchnerismo, recurriendo para ello a la dimensión de "contenidos", que apunta a la innovación de ideas en la vida política y en la tradición peronista, y a la dimensión de "instrumentos", que busca ver si el kirchnerismo se ha comportado como una "facción" o ha buscado nuevos mecanismos de confluencia y diálogo político.

La sección "Actores Sociales" recoge investigaciones referidas al heterogéneo conjunto de interlocutores del kirchnerismo. Bonvecchi muestra cómo la relación con los empresarios evolucionó desde 2003 llevando a una "reconfiguración de la relación Estado-empresarios bajo el formato de un capitalismo selectivo". Por su parte, Etchmendy trabaja en su capítulo el sindicalismo (peronista) para el período 2003-2011. Como continuación de sentidos con el capítulo anterior se plantea aquí un "neocorporativismo segmentado". Los movimientos sociales son retomados en el capítulo de Mauro y Rossi para analizar el diálogo-conflicto con el kirchnerismo. En este caso se abordan piqueteros, movimiento de DDHH, las marchas por la inseguridad y por el problema ambiental, así como la protesta "del campo". Se remarca el protagonismo de tales actores sociales, y cómo fue posible para éstos el acceso a canales estatales. Otro de los actores relevantes son los medios de comunicación, cuya relación con el kirchnerismo es analizada por Kitzberger. Como se señala en otras partes del libro, la crisis del campo va a ser un parteaguas en esta cuestión. Así un primer período (confrontación discursiva con políticas pragmáticas) va de 2003 a 2008, iniciándose a partir de entonces la transformación del discurso en "radicalización contrahegemónica y reformista".

La última sección, "Políticas Públicas", reúne trabajos sobre diversas áreas de acción pública, articulados por la pregunta acerca de la novedad del kirchnerismo en las mismas. En ellos uno de los temas es el del régimen electoral. Alessandro se dedica a analizar el proceso de sanción de la Ley 26.571, enriqueciendo su relato con el testimonio de su participación en los "diálogos" convocados por el Ministerio del Interior para el armado de la reforma en cuestión. Asimismo Scherlis analiza la legislación electoral, pero desde la mirada de la evolución del proyecto político kirchnerista, profundizando en las estrategias que aplicaron sobre la legislación electoral y las interpretaciones a las que apelaron durante las elecciones 2005, 2007 y 2009.

La relación entre gobierno nacional y gobiernos subnacionales es retomada por Lodola como una política pública distintiva, centrándose en las instituciones fiscales, la política tributaria y la política financiera. Al igual que en Gervasoni, el énfasis cae sobre la capacidad del gobierno nacional de redistribuir los recursos (selectivamente). En cuanto a otras políticas, Repetto aborda el análisis de las políticas sociales de transferencia de ingresos, enfatizando cómo en ello "se conjugaron virtuosamente un conjunto de factores permitiendo a los dos gobiernos, 
mediante un uso inteligente de las oportunidades políticas y el margen fiscal, avanzar en cambios relevantes que dejan, sin embargo, retos a afrontar". La política militar y la de defensa también experimentaron importantes cambios durante el kirchnerismo. Acorde a Battaglino, el relanzamiento de los juicios por las violaciones de los $\mathrm{DDHH}$, las medidas de fortalecimiento del gobierno político de la defensa y el renovado interés político por los temas de defensa nacional ilustran un giro hacia una lógica de "equilibrio". El apartado de las políticas públicas concluye con el capítulo de Llenderrozas dedicado al análisis de la política exterior kirchnerista. Las reflexiones llevan a conceptualizar la misma como "un estilo de posicionamiento internacional más que como una política exterior consistente". Se destaca la concentración de la decisión en manos del Presidente, el bajo rol de la cancillería, y la priorización de los asuntos internos.

Las conclusiones están a cargo de Murillo, quien realiza una síntesis de los puntos analizados sobre una matriz que entrecruza estabilidad y aplicación o imposición normativa para medir la dimensión de la fortaleza institucional. El resultado es la apreciación de los "grises", de las realidades heterogéneas, que han existido sobre la misma a lo largo de ambas gestiones kirchneristas.

En su conjunto los capítulos reseñados ofrecen una inteligente y completa respuesta a la inquietud inicial. Las reflexiones en muchos casos son productos de otras investigaciones de mayor alcance. Resulta frecuente encontrar en ellas diversos indicadores empíricos acompañando los argumentos vertidos. Fortaleciendo tal aspecto, el apartado final de Zelaznik, ofrece al lector un conjunto de datos claves (electorales, partidarios y legislativos) referidos al sistema político argentino.

A modo de cierre, es preciso hacer una referencia a la pregunta planteada acerca de qué perspectivas se avizoran, cuáles son los "asuntos pendientes" de las administraciones kirchneristas. En tal sentido se menciona la transformación del crecimiento en desarrollo sostenido, o bien la construcción de una nueva matriz federal de política social y un cambio significativo en los patrones de política pública en cuanto a la debilidad institucional, entre otros.

Se trata así de un libro de análisis político central para comprender la coyuntura argentina actual, que abre las puertas a numerosos debates al tiempo que permite tomar cada uno de los temas abordados como puntapiés o insumos para mayores investigaciones. 